\title{
An Unusual Developmental Profile of Salla Disease in a Patient with the SallaFIN Mutation
}

\author{
Liisa E. Paavola, ${ }^{1,2}$ Anne M. Remes, ${ }^{3,4}$ Pirkko H. Sonninen, ${ }^{5}$ Vesa V. Kiviniemi, ${ }^{6}$ \\ Tapio T. Korhonen, ${ }^{7}$ and Kari Majamaa ${ }^{1,2}$ \\ ${ }^{1}$ Department of Neurology, Oulu University Hospital, P.O. Box 20, 90029 Oulu, Finland \\ ${ }^{2}$ Department of Clinical Medicine, Neurology, University of Oulu, P.O. Box 5000, 90014 Oulu, Finland \\ ${ }^{3}$ Department of Neurology, Institute of Clinical Medicine, University of Eastern Finland, P.O. Box 1627, 70211 Kuopio, Finland \\ ${ }^{4}$ Department of Neurology, Kuopio University Hospital, P.O. Box 1777, 70211 Kuopio, Finland \\ ${ }^{5}$ Medical Imaging Centre, Turku University Hospital, P.O. Box 52, 20521 Turku, Finland \\ ${ }^{6}$ Department of Radiology, Oulu University Hospital, P.O. Box 20, 90029 Oulu, Finland \\ ${ }^{7}$ Division of Psychology, Department of Behavioural Sciences and Philosophy, University of Turku, 20014 Turku, Finland
}

Correspondence should be addressed to Liisa E. Paavola, liisa.paavola@neural.fi

Received 6 October 2012; Accepted 30 October 2012

Academic Editors: J. Lazareff and I. L. Simone

Copyright () 2012 Liisa E. Paavola et al. This is an open access article distributed under the Creative Commons Attribution License, which permits unrestricted use, distribution, and reproduction in any medium, provided the original work is properly cited.

Salla disease (SD) is a disorder caused by defective storage of free sialic acid and results from mutations in the SLC17A5 gene. Early developmental delay of motor functions, and later cognitive skills, is typical. We describe a developmental profile of an unusual homozygous patient, who harboured the SallaFIN (p.R39C) mutation gene. The study involved neurological examination, neuropsychological investigation, and brain imaging. The neurocognitive findings were atypical in comparison with other patients with the SallaFIN mutation. Interestingly, there was no deterioration in the patient's neurological condition during adulthood. Her neurocognitive skills were remarkably higher than those of other patients with a conventional phenotype of SD. Our results suggest that the phenotype of SD is broad. Unidentified genetic or environmental variation might explain the unique SD type of this case.

\section{Introduction}

Salla disease (SD; OMIM 604369) is a disorder characterised by defective storage of free sialic acid and belongs to the Finnish disease heritage [1]. However, sporadic cases of SD have been reported in many countries. The disease is caused by mutation of the SLC17A5 gene, which encodes a protein that transports sialic acid across the lysosomal membrane [2].

Salla disease affects the white matter by causing dysmyelination of the central nervous system and the peripheral nervous system. Magnetic resonance imaging studies have shown dysmyelination of the entire white matter of the cerebrum [3]. Cerebellar involvement has also been reported [4]. Hypoplasia of the corpus callosum is a typical finding in patients with SD. A conventional subtype and a severe subtype of the disease have been identified [5].
In neurocognitive terms, SD impacts nonverbal performance more than linguistic ability [6]. The common features related to nonverbal learning disabilities are associated with dysmyelination of the white matter. Another typical pattern seen in patients with SD is severe motor disability. After the second decade of life, the decline in motor skills is usually more pronounced than that in cognitive function. All affected individuals are intellectually disabled, but the level of cognitive and motor disabilities varies notably among patients with SD.

It is estimated that $95 \%$ of Finnish patients with SD are homozygous for the SallaFIN mutation: the p.R39C allele of the SLC17A5 gene [7]. Only a few patients are compound heterozygotes. These patients harbour the SallaFIN mutation in one allele of SLC17A5 and a different mutation in the other. Compound heterozygotes have a more severe phenotype than homozygotes [5]. Here we describe a patient with 
the homozygous SallaFIN mutation. Her neurocognitive development is unusual when compared with that of other patients with the same phenotype.

\section{Case Presentation}

The proband is a 30 -year-old woman. She was born after an uneventful pregnancy at full term. Her parents were nonconsanguineous. SD was diagnosed at 3 years of age on the basis of clinical symptoms and increased level of free sialic acid in the urine.

The patient's development during the first year of life was relatively normal, but crawling was unstable and muscular hypotonia and nystagmus were noticed. The patient spoke her first words at 1 year of age and her first sentences at 2 years of age. She learned to walk by 1.5 years of age, but her gait and balance were abnormal. At 3 years of age, her cognitive development was assessed as normal, except for mild slowness and clumsiness when performing fine motor skills. The followup evaluations showed mild delays in motor tasks, eye-hand coordination, and concentration. Her verbal development was slightly delayed, and verbal dyspraxia was reported. At 6 years of age, the developmental delay was approximately 2 years.

Inattentiveness, hyperactivity, and problems with sleep were reported during childhood. The patient also had problems with balance and body awareness. Ataxic symptoms were prominent in childhood, but improved during the teenage years.

During her school years, the neurocognitive development fluctuated notably. Verbal performance was consistently better than visual performance or fine motor skills. Intellectual disability was considered to be mild.

At the age of 12 years, the patient's verbal skills, as assessed using the Wechsler Intelligence Scale for Children-R test [8], were at the level of a 7 year old, and her performance skills varied between those typical of a child of 5 years 6 months and 6 years 6 months of age. Two years later, her verbal skills had improved. At 14 years of age, no progression was noted in the neurocognitive deficits. The developmental age of the patient varied between 4 and 8 years, and her verbal skills were notably better than her motor and visual abilities.

2.1. Neurological Examination. At the age of 30 years, the proband was living alone with support. She was a social person, keen on the arts and team sports. She was $157 \mathrm{~cm}$ in height and weighed $56 \mathrm{~kg}$. She was taking no medications. On examination, auscultation of the heart and lungs was unremarkable, her blood pressure was $114 / 74 \mathrm{mmHg}$, and the electrocardiogram was normal. Her facial features were slightly coarse. The proband could walk without aid, but both legs were in a pes planus position. When walking, she had some athetotic movements in her upper extremities. Muscle strength and skin sensation were normal, tendon reflexes were symmetrical and normal, and the plantar responses were in flexion. Both Achilles tendons were slightly shortened and there was mild spasticity in both legs. Neurological examination revealed only mild ataxia.
There was mild instability in the Romberg test and the patient was unable to stand with her eyes closed. There was no ataxia or dysmetria shown by coordination tests, but her hand movements were clumsy. She suffered from marked myopia and used six dioptre corrective lenses. Clear outward strabismus was seen in her right eye. However, the eye movements were normal and nystagmus was not detected. The neurological condition of the patient had not deteriorated during the previous 10 years.

There had been no deterioration of the patient's motor skills in adulthood. Her skills had improved with respect to balance, coordination of body movements, and reciprocal motor actions, as well as processing the sequences of movements. The speed of motor actions had become slightly slower during the last few years.

The electroencephalogram (EEG) was normal at 3 years of age, but showed mild generalized background abnormality with occasional spikes and sharp waves at the left temporoparieto-central region at 5 years of age. Quantitative EEG was normal at 15 years of age. There was no history of epileptic seizures, but symptoms that resembled the startle reflex were noticed in response to sudden noises.

2.2. Neurocognitive and Motor Development. The methods that were used for neuropsychological evaluation of the patient are presented in Table 1. Her developmental age, as assessed by Wechsler Intelligence Scale for Children-III [9] at the age of 30 years, was 7 years 9 months for the verbal scale and 5 years 4 months for the perceptual scale. Her neurocognitive performance was remarkably better than those of other patients with the conventional type of SD and the SallaFIN mutation [6]. Other patients with SD $(n=$ 37) have been evaluated using the Bayley Scales of Infant Development-II [14], because the tasks that are used in the Wechsler children's tests were too demanding.

There was a slowing in visuomotor speed as well as eyehand coordination during the followup of our patient after her teenage years. Visual reasoning and spatial orientation were mildly delayed, and the visuoconstructive skills were diminished. However, verbal skills had improved. Repetition of nonsense words and oromotor sequences were difficult for the proband because of verbal and oral dyspraxia, but the proband was able to learn and repeat long, logical stories. She had difficulties with time orientation.

Motor problems were evident but the symptoms had not progressed during the followup. The proband was able to walk on the toes and sides of the feet, but the forward tandem walk was insecure, and motor persistence and motor coordination were clumsy. Static cerebellar tests were performed quite well, with only slight problems with balance. Two of the dynamic cerebellar tests-finger-tothumb tapping and toe tapping - were performed slowly but correctly. Visuomotor deficits were evident, but the proband managed the test of basic functional mobility quite well.

2.3. Brain Imaging. Brain imaging performed at 15 years of age showed dysmyelination. The corpus callosum was hypoplastic, but the cerebellum, pons, and the proximal part 
TABLE 1: Neuropsychological evaluation of the proband at 30 years of age.

\begin{tabular}{|c|c|c|c|c|}
\hline Full name of the test & Abbreviation & Reference & $\begin{array}{l}\text { The domain of the test/chosen } \\
\text { parts }\end{array}$ & Results $^{\mathrm{a}}$ \\
\hline \multirow{2}{*}{$\begin{array}{l}\text { Wechsler Intelligence Scale for } \\
\text { Children-III }\end{array}$} & \multirow{2}{*}{ WISC-III } & \multirow{2}{*}{ Wechsler (1991) [9] } & $*$ Verbal skills & 1 \\
\hline & & & $*$ Perceptual skills & 1 \\
\hline \multirow{3}{*}{$\begin{array}{l}\text { Children's Neuropsychological Test } \\
\text { Battery }\end{array}$} & \multirow{3}{*}{ NEPSY } & \multirow{3}{*}{ Korkman et al. (1997) [10] } & ${ }^{*}$ Comprehension of instructions & 0 \\
\hline & & & $*$ Oromotor sequences & 2 \\
\hline & & & ${ }^{*}$ Repetition of nonsense words & 2 \\
\hline $\begin{array}{l}\text { Physical and Neurological Examination } \\
\text { of Soft Signs }\end{array}$ & PANESS & Denckla (1985) [11] & Corpus callosum dysfunction & 2 \\
\hline Static and Dynamic Cerebellar Tests & Cerebellar tests & Fawcett et al. (2001) [12] & Dysfunction of cerebellum & 1 \\
\hline Timed Up-and-Go-test & TUG-test & Williams et al. (2005) [13] & Basic and functional mobility & 1 \\
\hline
\end{tabular}

${ }^{\mathrm{a}} 0=$ among average, 1 = mild deficits, 2 = severe deficits.

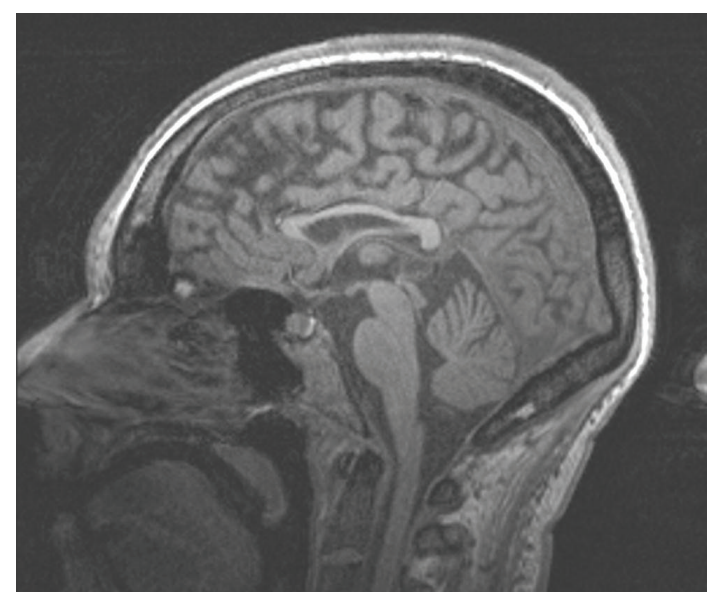

(a)

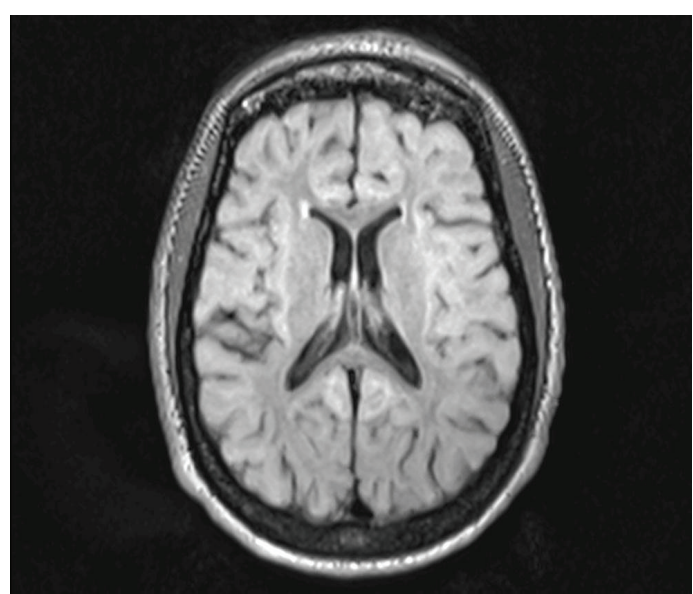

(b)

FIGURE 1: Hypoplastic corpus callosum in magnetic resonance imaging of the patient. (a) sagittal plane; (b) axial plane.

of the cervical cord were normal. There was no enlargement of the ventricles or signs of cortical atrophy. The MRI findings at 30 years of age were mild (Figure 1).

\section{Discussion}

The typical neurocognitive profile of SD consists of a lower level of nonverbal performance as compared with linguistic skills. Findings related to nonverbal learning disabilities have been outlined [6]. Herein, we have described an unusual developmental profile of SD in a patient with the SallaFIN mutation. Her neurocognitive development differs from that of other patients with SD of the conventional subtype who carry the SallaFIN mutation. Her neurological condition has remained fairly constant during adulthood. Only mild progression of the symptoms related to her neurocognitive skills has been seen. The MRI findings showed that the ventricles were of normal size; the corpus callosum was thin, but there was no cortical atrophy.

The patient has received regular physiotherapy and has participated actively in sports since childhood. The benefits to the brain of physical activity, which include anatomical, functional, and molecular changes, have been documented [15]. Physical activity also affects the health of the neural network and on the capacity to process information.

Unknown genetic and environmental variation might explain the unique SD type of the proband. The heterogeneity of the severity and progression of SD is a challenge for diagnostic work and rehabilitation with both children and adult patients.

\section{Acknowledgments}

The study was supported by Finnish Brain Foundation, Finnish Cultural Foundation, and Maire Taponen Foundation.

\section{References}

[1] P. Aula, S. Autio, and K. O. Raivio, "'Salla disease'. A new lysosomal storage disorder," Archives of Neurology, vol. 36, no. 2, pp. 88-94, 1979.

[2] F. W. Verheijen, E. Verbeek, N. Aula et al., "A new gene, encoding an anion transporter, is mutated in sialic acid storage diseases," Nature Genetics, vol. 23, no. 4, pp. 462-465, 1999. 
[3] P. Sonninen, T. Autti, T. Varho, M. Hämäläinen, and R. Raininko, "Brain involvement in Salla disease," American Journal of Neuroradiology, vol. 20, no. 3, pp. 433-443, 1999.

[4] T. Varho, M. Komu, P. Sonninen et al., "A new metabolite contributing to $\mathrm{N}$-acetyl signal in $1 \mathrm{H}$ MRS of the brain in Salla disease," Neurology, vol. 52, no. 8, pp. 1668-1672, 1999.

[5] T. T. Varho, L. E. Alajoki, K. M. Posti et al., "Phenotypic spectrum of Salla disease, a free sialic acid storage disorder," Pediatric Neurology, vol. 26, no. 4, pp. 267-273, 2002.

[6] L. Alajoki, T. Varho, K. Posti, P. Aula, and T. Korhonen, "Neurocognitive profiles in Salla disease," Developmental Medicine and Child Neurology, vol. 46, no. 12, pp. 832-837, 2004.

[7] N. Aula, P. Salomäki, R. Timonen et al., "The spectrum of SLC17A5 mutations resulting in free sialic acid-storage diseases indicates some genotype-phenotype correlation," American Journal of Human Genetics, vol. 67, no. 4, pp. 832840,2000

[8] D. Wechsler, Wechsler Intelligence Scale For Children-Revised. A Finnish Adaptation, Psykologien Kustannus Oy, Helsinki, Finland, 1984.

[9] D. Wechsler, Wechsler Intelligence Scale For Children-Third Edition, The Psychological Corporation, San Antonio, Tex, USA, 1991.

[10] M. Korkman, U. Kirk, and S. L. Kemp, NEPSY. Neuropsychological Assessment of Children, The Psychological Corporation, San Antonio, Tex, USA, 1997.

[11] M. B. Denckla, "Revised neurological examination for subtle signs," Psychopharmacology Bulletin, vol. 21, no. 4, pp. 773800, 1985.

[12] A. J. Fawcett, R. I. Nicolson, and F. Maclagan, "Cerebellar tests differentiate between groups of poor readers with and without IQ discrepancy," Journal of Learning Disabilities, vol. 34, no. 2, pp. 119-135, 2001

[13] E. N. Williams, S. G. Carroll, D. S. Reddihough, B. A. Philips, and M. P. Galea, "Investigation of the timed "Up \& Go" test in children," Developmental Medicine and Child Neurology, vol. 47, no. 8, pp. 518-524, 2005.

[14] N. Bayley, Bayley Scales of Infant Development, BSID, Psychological Corporation, New York, NY, USA, 2nd edition, 1993.

[15] C. W. Cotman, N. C. Berchtold, P. A. Adlard, and V. M. Perreau, "Exercise and brain," in Molecular and Cellular Exercise Physiology, F. C. Mooren and K. Völker, Eds., pp. 331341, Human Kinetics, Stanningley, UK, 2005. 


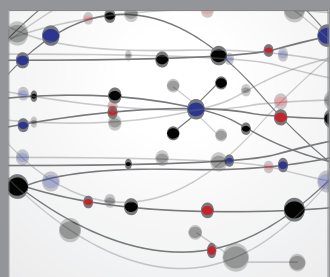

The Scientific World Journal
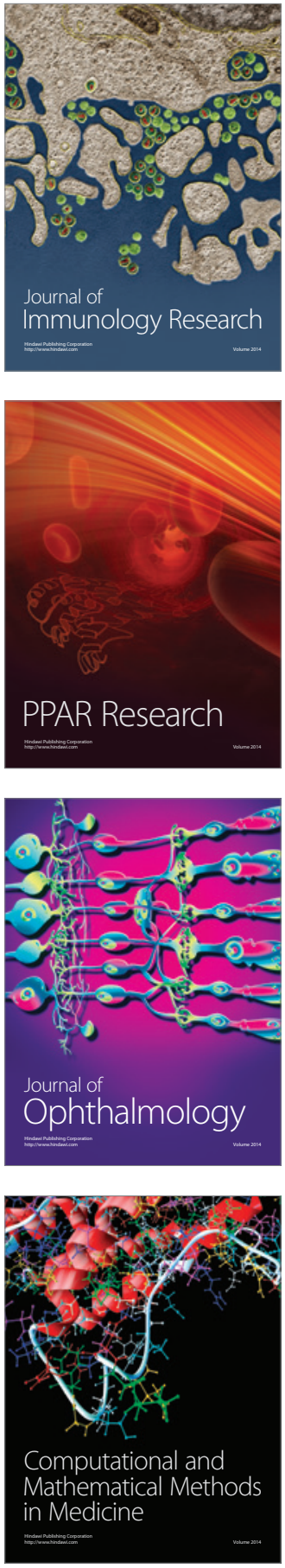

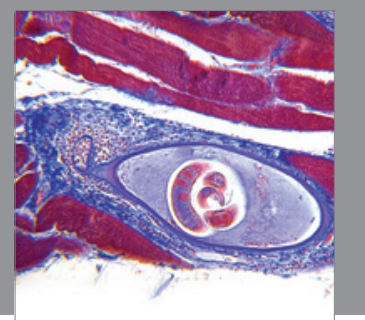

Gastroenterology

Research and Practice
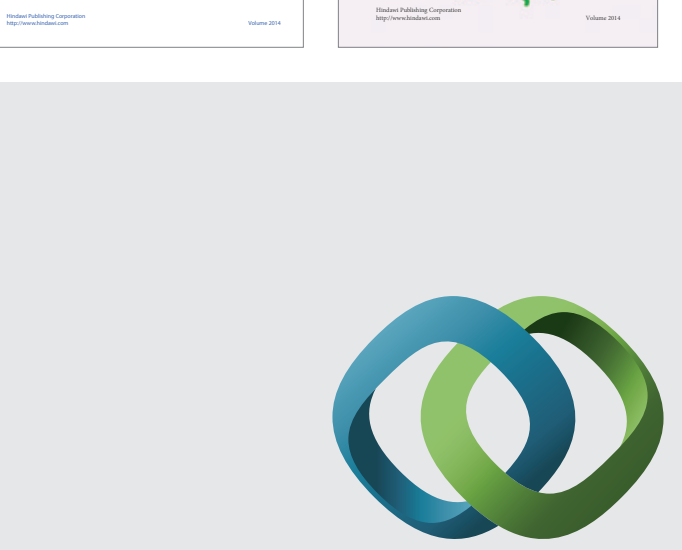

\section{Hindawi}

Submit your manuscripts at

http://www.hindawi.com
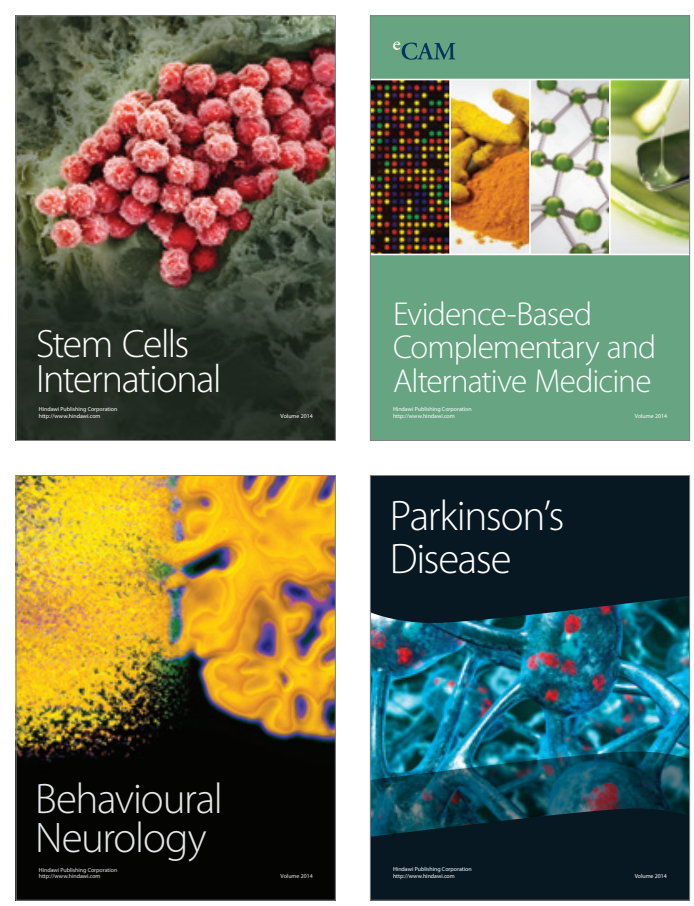

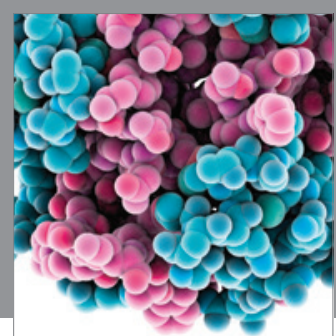

Journal of
Diabetes Research

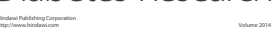

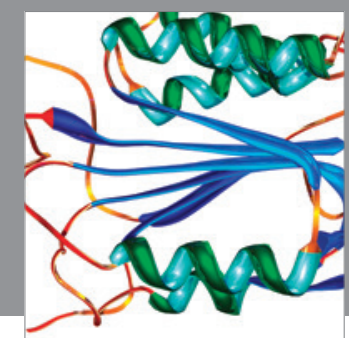

Disease Markers
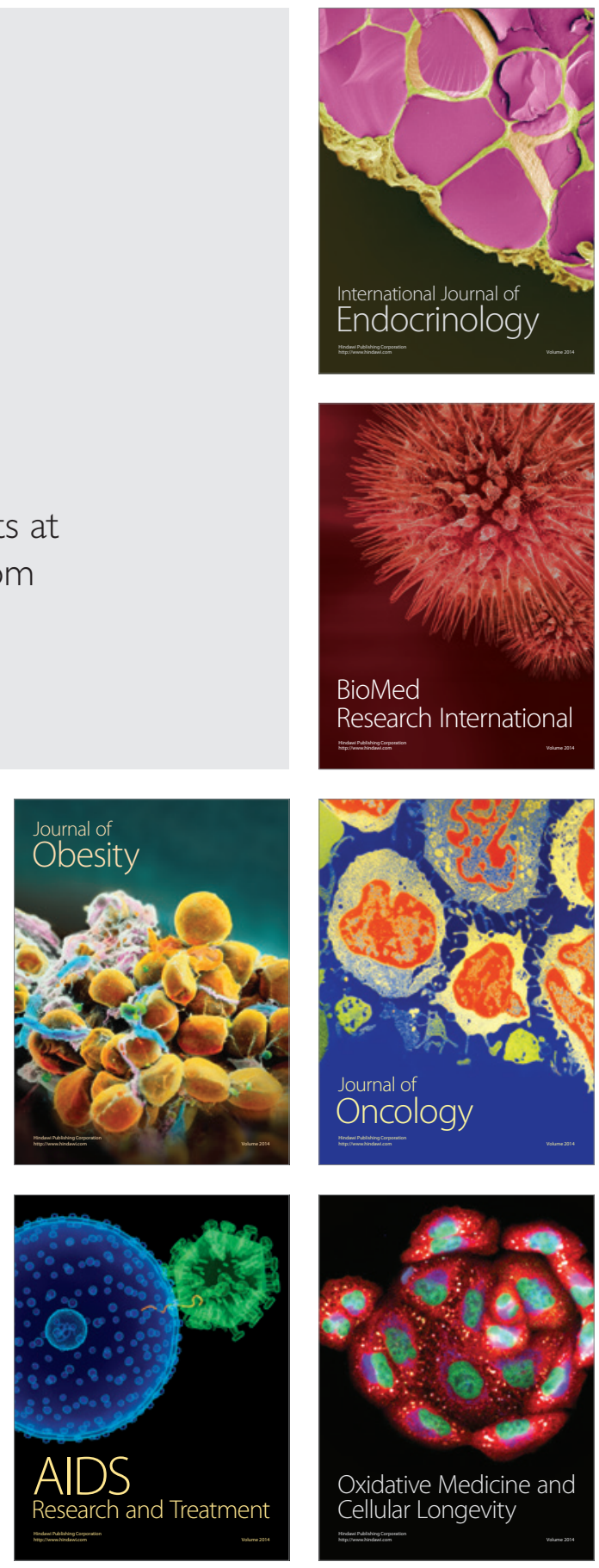